\title{
Study of decoherence in a system of superconducting flux-qubits interacting with an ensemble of electrons
}

\author{
M. Reboiro ${ }^{\mathrm{a}, *}$, O. Civitarese ${ }^{\mathrm{a}}$, R. Ramírez ${ }^{\mathrm{b}}$ \\ a IFLP, CONICET-Department of Physics, University of La Plata, C.c. 67 1900, La Plata, Argentina \\ ${ }^{\mathrm{b}}$ IFLP, CONICET-Department of Mathematics, University of La Plata, Argentina
}

\section{H I G H L I G H T S}

- The degree of coherence in a hybrid system, composed of superconducting flux qubits and an electron ensemble, is analysed.

- The time evolution of the hybrid system is solved exactly and discussed in terms of the reduced density matrix of each subsystem.

- It is shown that the initial state of the system evolves to a stationary squeezed state.

\section{A R T I C L E I N F O}

\section{Article history:}

Received 14 October 2016

Accepted 25 January 2017

Available online 30 January 2017

\section{Keywords:}

Hybrid systems

Dissipation

Spin-squeezing

Decoherence

\begin{abstract}
A B S T R A C T
The degree of coherence in a hybrid system composed of superconducting flux-qubits and an electron ensemble is analysed. Both, the interactions among the electrons and among the superconducting flux-qubits are taken into account. The time evolution of the hybrid system is solved exactly, and discussed in terms of the reduced density matrix of each subsystem. It is seen that the inclusion of a line width, for the electrons and for the superconducting flux-qubits, influences the pattern of spin-squeezing and the coherence of the superconducting flux qubits.
\end{abstract}

(c) 2017 Elsevier Inc. All rights reserved.

\footnotetext{
* Corresponding author.

E-mail addresses: reboiro@fisica.unlp.edu.ar (M. Reboiro), osvaldo.civitarese@fisica.unlp.edu.ar (O. Civitarese).
} 


\section{Introduction}

Controlled manipulation of coherent quantum systems is a crucial step for quantum information technology [1-6]. Considerable efforts have been devoted, both experimentally and theoretically, to understand the role of coherence in different physical systems. Among other schemes, the excitation of quantum dots has been shown to provide discrete atomic-like transitions which can be manipulated using optical pulses $[7,8]$. In the same direction, the dynamics of spontaneous generation of coherence and photonspin-qubit entanglement in a $\Lambda$-system has been discussed in [9]. Other promising candidates for the realization of quantum processing devices are hybrid systems [10]. An example of this type of architecture has been proposed in [11]. It consists of a superconducting flux-qubit coupled to electrons in the form of nitrogen-vacancy (NV) colour centres in diamond [11-13]. The Authors of Ref. [11] have demonstrated the existence of a strong coherent-coupling in these systems. They have observed the coherent exchange (transfer) of a single quantum of energy. These developments can be seen as first steps towards the design of long-lived quantum memories. Further studies of non-equilibrium phases in flux-qubits and NV centres have been reported in [14]. The theoretical framework in which these studies have been performed is modelled by a Jaynes-Cummings-lattice, both in the strong and weak coupling limits. In the same line of proposals, in Ref. [15] it is reported the study of a hybrid quantum system consisting of spin ensembles and superconducting flux qubits, each spin ensemble is realized using the nitrogen-vacancy centres in a diamond crystal, and the nearestneighbour spin ensembles are effectively coupled via a flux-qubit. The analysis of the properties of these physical systems includes the study of the interplay between entanglement, squeezing, and decoherence [16-20].

Recently, the study of quantum properties as a function of time, in open systems, has received renewed attention, particularly in relation to the properties of entanglement [21-26]. In Ref. [21] it is analysed the behaviour of a system of spins, which are assumed to have a finite lifetime, interacting through a Lipkin type [27,28]. It is shown that the system amplifies the pattern of spin squeezing when the line width of the spin states are taken into account. Similar results have been found in [22]. The Authors of [22] have investigated the enhancement of spin squeezing in a non-Hermitian one-axis twisting model and its persistence in time [29]. The Authors of [23,24] have reexamined the steady-state behaviours for a spin ensemble in diamond nanostructures by exerting a controllable microwave field. The study of an open hybrid system has been proposed in [25], where it is reported the formation of a robust state against noise due to the coupling of a NV centre to a two level short-lived system.

We have taken the findings of [21-25] to investigate the effects of coherence in the hybrid system of a superconducting flux-qubit and electrons [11,14]. Our starting point was the diagonalization of the Hamiltonian of the system, and the calculation of the reduced density-matrix of the qubit and electron sectors. Then, we have calculated the time evolution of the squeezing parameters, to correlate the time dependence of the non-diagonal terms of the reduced density-matrix, of each sector, with the time dependence of these parameters. The details of the formalism are presented in Section 2. The results of the calculations are presented and discussed in Section 3. It is shown that the pattern of revival of the spin-observables of the superconducting flux-qubit is strongly affected by the interactions. This finding complements the results of [14] where the onset of coherence was not considered but suggested to be important. Our conclusions are drawn in Section 4.

\section{Formalism}

Let us consider a hybrid system, combination of an array of superfluid flux qubits (SFQ) and an ensemble of electrons, in the form of nitrogen-vacancy (NV) centres in diamond [14]. The study of this type of architecture is motivated by its potential use in quantum computing design. The flux qubits can be viewed as the quantum-computing processor and the NV centres, due to their large coherence time [30-32], can be implemented as the quantum memory [12]. From the experimental point of view, arrays of flux qubits with tunable coupling strength between individual qubits have been realized using a SQUID or ancilla flux qubit [33-38]. The physics of coupling SFQ and NV ensembles has 
been well established, both theoretically and experimentally [11,12]. The spin-qubit interaction is mediated by the magnetic field that stems from the persistent currents of the qubits.

The Hamiltonian of a hybrid system can be written as [11,12,39]

$$
H_{\text {hybrid }}=H_{F q}+H_{S}+H_{\text {int }-q s} \text {. }
$$

The term $H_{F q}$ of Eq. (1) is the Hamiltonian of the superconducting flux-qubits. In the basis of clockwise and anticlockwise qubit-persistent-currents [11,14,40-42], it reads

$$
H_{F q}=\frac{1}{2} \sum_{k=1}^{N_{q b}}\left(\Delta_{k} s_{X, k}+\epsilon_{k} S_{z, k}\right)+\sum_{\substack{k, k^{\prime} \\ k \neq k^{\prime}}}^{N_{q b}} J_{k, k^{\prime}} S_{z, k} S_{z, k^{\prime}},
$$

where $\left\{s_{x, k}, s_{y, k}, s_{z, k}\right\}$ are Pauli spin-1/2 operators. The parameter $\epsilon_{k}$ is the energy bias, $\epsilon_{k}=$ $2 I_{p, k}\left(\Phi_{e x, k}-3 \Phi_{0} / 2\right), I_{p, k}$ is the persistent current in a qubit at site $k, \Phi_{e x, k}$ is the external flux threading the qubit loop, $\Phi_{0}=1 /(2 e)$ is the flux-quantum and $\Delta_{k}$ is the tunnel splitting. Here, $J_{k, k^{\prime}}$ denotes the coupling strength between qubits in the sites $k$ and $k^{\prime}$, respectively.

An NV centre has a ground state with spin 1 and a zero-field splitting $D=2.88 \mathrm{GHz}$ between the $|1,0\rangle$ and $|1, \pm 1\rangle$ states. If an external magnetic field, along the crystalline axis of the NV centre, is applied an additional Zeeman splitting between $|1, \pm 1\rangle$ sub-levels occurs. Then, it is possible to isolate the subsystem form by $|1,0\rangle$ and $|1,1\rangle$, so that the NV centre can be modelled by a two-level system [39,43]. The effective spin-spin interaction [44-46] of the NV-ensemble Hamiltonian, $H_{S}$ of Eq. (1), can be written as

$$
H_{S}=D S_{z}^{2}+E\left(S_{x}^{2}-S_{y}^{2}\right)
$$

where the operators $\left\{S_{x}, S_{y}, S_{z}\right\}$ are the collective Pauli spin-operators, components of the total spin, S, of the electrons. This sector of the Hamiltonian consists of a one-twist term (OAT) [29,44,45], $D S_{z}^{2}$, responsible for the squeezing pattern, and of a Lipkin-type interaction $E\left(S_{x}^{2}-S_{y}^{2}\right)$, being $E$ the ground state strain-induced splitting coefficient $[28,27,47,48]$.

The interaction among the SFQ and the NV ensemble is favoured by the similarity in the energy splitting. The two states of the SFQ are typically separated by a few $\mathrm{GHz}$, while NV centres zero-field splitting $D=2.88$ [GHz]. Following the works of $[11,12,39]$, we shall introduce the coupling of the SFQ with the NV spin-ensemble, that is the term $H_{\text {int-qs }}$ of Eq. (1):

$$
H_{\text {int }-q s}=\frac{1}{2} \sum_{k=1}^{N_{q b}} g_{k} S_{z, k} S_{x} .
$$

The coupling strength $g_{k}$ is proportional to the magnitude of the qubit field at the spin location.

The gap-tunable flux-qubit Hamiltonian, $H_{F q}$ of Eq. (2), is diagonalized by the transformation

$$
\left(\begin{array}{l}
s_{z, k} \\
s_{x, k} \\
s_{y, k}
\end{array}\right)=\left(\begin{array}{ccc}
\cos \alpha_{k} & \sin \alpha_{k} & 0 \\
-\sin \alpha_{k} & \cos \alpha_{k} & 0 \\
0 & 0 & 1
\end{array}\right)\left(\begin{array}{l}
\sigma_{z, k} \\
\sigma_{x, k} \\
\sigma_{y, k}
\end{array}\right) .
$$

Both set of operators, $\left\{\sigma_{x, k}, \sigma_{y, k}, \sigma_{z, k}\right\}$ and $\left\{s_{x, k}, s_{y, k}, s_{z, k}\right\}$, obey the $s u(2)$ algebra. The parameter $\alpha_{k}$ of the transformation is related to parameters of the qubit flux by $\cos \alpha_{k}=\epsilon_{k} / E_{q b, k}, \sin \alpha_{k}=-\Delta_{k} / E_{q b, k}$, and $E_{q b, k}=\sqrt{\epsilon_{k}^{2}+\Delta_{k}^{2}}$.

In terms of the new operators $\sigma_{i}(i=x, y, z), H_{F q}$ and $H_{\text {int-qs }}$ can be written as

$$
\begin{aligned}
H_{1}= & \sum_{k} \frac{1}{2} E_{q b, k} \sigma_{z, k}+\sum_{\substack{k, k^{\prime} \\
k \neq k^{\prime}}}^{N_{q b}} J_{k, k^{\prime}}\left(\cos \alpha_{k} \sigma_{z, k}+\sin \alpha_{k} \sigma_{x, k}\right) \\
& \times\left(\cos \alpha_{k^{\prime}} \sigma_{z, k^{\prime}}+\sin \alpha_{k^{\prime}} \sigma_{x, k^{\prime}}\right), \\
H_{2}= & \frac{1}{2} \sum_{k} g_{k}\left(\cos \alpha_{k} \sigma_{z, k}+\sin \alpha_{k} \sigma_{x, k}\right) S_{x} .
\end{aligned}
$$


We shall assume that the spins (electronic states) and the superconducting flux-qubits have a finite lifetime given by their line-widths $\gamma_{s}$ and $\gamma_{q b}$, respectively [10]. We shall model the corresponding term of the Hamiltonian as [21,49]

$$
H_{\gamma}=-\mathrm{i} \gamma_{s} S_{z}-\mathrm{i} \sum_{k=1}^{N_{q b}} \gamma_{q b, k} \sigma_{z, k} .
$$
like

Finally, the Hamiltonian of the hybrid system, $H$, is given by the sum of the terms (3), (6) and (7),

$$
H=H_{1}+H_{2}+H_{S}+H_{\gamma}
$$

and it can be diagonalized in the product basis

$$
\begin{aligned}
\left|N_{q b},\left\{k_{q b, j}\right\}, N_{S}, k_{S}\right\rangle & =\left|N_{q b},\left\{k_{q b, j}\right\}\right\rangle \otimes\left|N_{S}, k_{S}\right\rangle, \\
\left|N_{S}, k_{S}\right\rangle & =\mathcal{N}_{S} S_{+}^{k_{S}}|0\rangle_{S}, \\
\left|N_{q b},\left\{k_{q b, j}\right\}\right\rangle & =\mathcal{N}_{q b} \prod_{j=1}^{N_{q b}} \sigma_{+, j}^{k_{q b, j}}|0\rangle_{q b},
\end{aligned}
$$

where $S_{ \pm}=S_{x} \pm i S_{y}$. The label $k_{S}$ can run from 0 to the number of spins (electrons) of the system, $N_{S}$. Similarly, $\sigma_{ \pm, j}=\sigma_{x, j} \pm i \sigma_{y, j}$, and $k_{q b, j}=0$, 1 . We denote with $\left\{k_{q b, j}\right\}$ each of arrays of $N_{q b}$ superconducting qubits. The quantities $\mathcal{N}$ are normalization factors. Consequently, the model space has $2^{N_{q b}} \times\left(N_{s}+1\right)$ configurations.

\subsection{Time evolution}

Dissipative dynamics can be captured by introducing non-Hermitian descriptions. The Hamiltonian of an open quantum system consists formally of a first order interaction term describing the closed (isolated) system with discrete states and a second-order term caused by the interaction of the discrete states via the common continuum of scattering states. Using the Feshbach [49] projection operator formalism, the solution of the Schrödinger equation in the whole function space (with discrete as well as scattering states, and an Hermitian Hamilton operator $\mathrm{H}$ ) can be represented in the interior of the localized part of the system in the set of eigenfunctions of an effective non-Hermitian Hamiltonian.

We shall follow the work of [50], to address the time evolution of the non-Hermitian effective Hamiltonian of Eq. (8). We shall construct a bi-orthonormal basis, $\left\{\left|\bar{\Phi}_{\alpha}\right\rangle,\left|\widetilde{\Phi}_{\beta}\right\rangle\right\}$, from

$$
\begin{aligned}
H\left|\widetilde{\Phi}_{\alpha}\right\rangle & =E_{\alpha}\left|\widetilde{\Phi}_{\alpha}\right\rangle, \\
\left\langle\bar{\Phi}_{\alpha}\right| H^{\dagger} & =\left\langle\bar{\Phi}_{\alpha}\right| E_{\alpha}^{*} .
\end{aligned}
$$

The states of the bi-orthonormal basis obey the condition

$$
\left\langle\bar{\Phi}_{\alpha} \mid \widetilde{\Phi}_{\beta}\right\rangle=\delta_{\alpha \beta} \text {. }
$$

Let us consider a general initial state, $|I\rangle$. In terms of the eigenvectors of $H$ the initial state can be written as

$$
\widetilde{I}\rangle=\sum_{\alpha} \widetilde{c}_{\alpha}\left|\widetilde{\Phi}_{\alpha}\right\rangle
$$

As $H$ is non-Hermitian, we have $(\hbar=1)$

$$
\begin{aligned}
& \tilde{I}(t)\rangle=\mathrm{e}^{-i H t}|\tilde{I}\rangle=\sum_{\alpha} \widetilde{c}_{\alpha} \mathrm{e}^{-i E_{\alpha} t}\left|\widetilde{\Phi}_{\alpha}\right\rangle, \\
& \langle\bar{I}(t)|=\langle\bar{I}| \mathrm{e}^{i H^{\dagger} t}=\sum_{\alpha} \bar{c}_{\alpha} \mathrm{e}^{i E_{\alpha}^{*} t}\left\langle\bar{\Phi}_{\alpha}\right| .
\end{aligned}
$$

Thus, the mean value of an operator 0 , at time $t$, is evaluated as

$$
\langle\bar{I}(t)|O| \tilde{I}(t)\rangle \text {. }
$$




\subsection{Initial condition}

To study the time evolution of the states and observables described by the previous section we follow the formalism presented in Ref. [51]. We shall assume that the initial state of the hybrid system is a direct product of the electron ensemble initial state and of the initial state of the superconducting qubits

$$
|I(0)\rangle=|I\rangle_{q b} \otimes|I\rangle_{S} .
$$

For the electron ensemble initial state we shall consider a coherent spin-states of the form

$$
|I\rangle_{S}=N_{S} \mathrm{e}^{z_{S} S_{+}}|0\rangle,
$$

with $z_{S}=-\mathrm{e}^{-i \phi_{S}} \tan \left(\theta_{S} / 2\right)$, where the angles $\left(\theta_{S}, \phi_{S}\right)$ define the direction $\vec{n}_{S}=\left(\sin \theta_{S} \cos \phi_{S}, \sin \theta_{S}\right.$ $\left.\sin \phi_{S}, \cos \theta_{S}\right)$, such that $\vec{S} \cdot \vec{n}_{S}|I\rangle_{S}=-S|I\rangle_{S}$, with $S=N_{S} / 2$ [52].

For the initial state of the superconducting qubits, we shall consider a particular state of the form $\left|N_{q b},\left\{k_{q b}\right\}\right\rangle$ (Eq. (9)).

\subsection{Spin-squeezing parameter}

Spin-squeezed-states are quantum-correlated states with reduced fluctuations in one of the components of the total spin. Let us consider a general quasi-spin operator, $\mathbf{T}$, with components $\left\{T_{x}, T_{y}, T_{z}\right\}$. Following the work of Ueda and Kitagawa [29], we shall define a set of orthogonal axes $\left\{\mathbf{n}_{\mathbf{x}^{\prime}}, \mathbf{n}_{\mathbf{y}^{\prime}}, \mathbf{n}_{\mathbf{z}^{\prime}}\right\}$, such that $\mathbf{n}_{\mathbf{z}^{\prime}}$ is the unitary vector pointing along the direction of the total spin $\langle\mathbf{T}\rangle$. We shall fix the direction $\mathbf{n}_{\mathbf{x}^{\prime}}$ by looking at the minimum value of $\left(\Delta T_{x^{\prime}}\right)^{2}$, consequently, we define the squeezing factor as

$$
\zeta_{x^{\prime}}^{2}=\frac{2\left(\Delta T_{x^{\prime}}\right)^{2}}{|\langle\mathbf{T}\rangle|} .
$$

Then, the state is squeezed if $\zeta_{x^{\prime}}^{2}<1$. So defined, the parameter of Eq. (18) is su(2) invariant [53]. For completeness, we shall define

$$
\zeta_{y^{\prime}}^{2}=\frac{2\left(\Delta T_{y^{\prime}}\right)^{2}}{|\langle\mathbf{T}\rangle|} .
$$

Clearly $\zeta_{x^{\prime}}^{2} \zeta_{y^{\prime}}^{2} \geq 1$

\subsection{Decoherence}

Interactions with the environment lead to a rapid cancellation of the off-diagonal terms of the local density matrix describing the probability of measurements. This effect is known as environmentinduced decoherence $[17,16]$. We shall analyse the behaviour of the off-diagonal terms of the reduced density matrix of a superconducting flux-qubit, by assuming that the environment is modelled by the electron ensemble.

The elements of the density matrix of the system read

$$
\rho_{\alpha, \beta}=\widetilde{c}_{\alpha} \bar{c}_{\beta} \mathrm{e}^{-\mathrm{i}\left(E_{\alpha}-E_{\beta}^{*}\right) t} .
$$

\section{Results and discussion}

In this section we shall present and discuss the results of the calculations, which have been performed by adopting the parameters given in Refs. $[11,14]$ for the qubit- and electron-sectors of the 


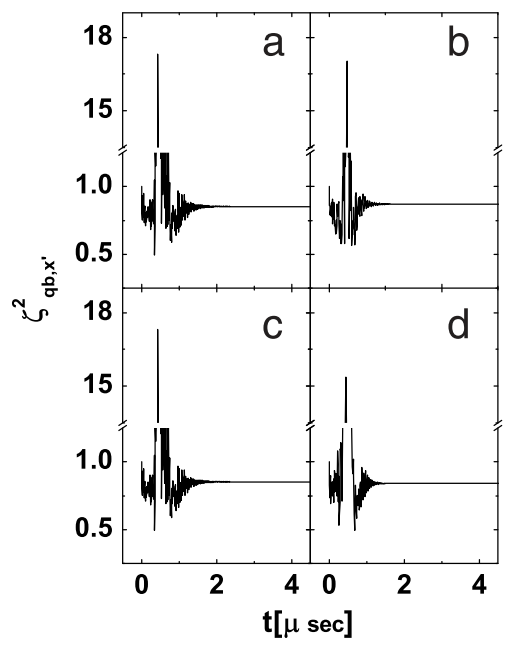

Fig. 1. Behaviour, as a function of time and in units of $\mu$ second, of the superconducting flux qubits squeezing parameter, $\zeta_{q b, x^{\prime}}^{2}$, in absence of coupling to the electron ensemble. In Insets (a), (b), (c) and (d), we have displayed the results obtained for systems with $N_{q b}=3,4,5$ and 6, respectively. The coupling constants of the Hamiltonian of Eq. (2), $H_{q b}$, were fixed at the values $\Delta_{k}=2.40[\mathrm{GHz}], \epsilon_{k}=1.6[\mathrm{GHz}]$, and the site-dependent coupling constant of the qubit-qubit interaction was fixed at the values $J_{k, k^{\prime}}=0.32 \times 2^{-\left|k^{\prime}-k\right|}[\mathrm{GHz}]$. The line-width was fixed at $\gamma_{q b}=4 \times 10^{-3}$ [GHz].

system. For the electron sector we have fixed the coupling constants to the values $D=2.88$ [GHz], $E=0.15[\mathrm{GHz}]$ and $\gamma_{s}=2 \times 10^{-5}[\mathrm{GHz}]$, the adopted value of $\gamma_{s}$ is with consistent a coherence time of the electron ensemble $T_{S} \gg 10$ [ $\left.\mu \mathrm{s}\right]$ [11,30-32]. For the superconducting flux-qubits we have fixed the values of the coupling constants to $\Delta_{k}=2.40[\mathrm{GHz}]$ and $\epsilon_{k}=1.6[\mathrm{GHz}]$, so that $E_{q b, k}=\sqrt{\epsilon_{k}^{2}+\Delta_{k}^{2}}=2.89[\mathrm{GHz}]$, with $k=1, \ldots, N_{q b}$ [33-38]. We have adopted a value of $\gamma_{q b}$ consistent with the time of coherence reported in [34] for a system of three superconducting flux qubits, that is $T_{q b}=2[\mu \mathrm{s}]$ and $\gamma_{q b}=4 \times 10^{-3}[\mathrm{GHz}]$. We shall assume that the initial state for the superconducting flux qubits is prepared as $\left|N_{q b},\left\{k_{q b, j}\right\}=\{1,1, \ldots, 1\}\right\rangle$ (Eq. (9)), and that the initial state for the electron ensemble is a coherent state with $\theta_{0}=\pi / 4$ and $\phi_{0}=0$.

In Figs. 1-3, we analyse the properties of a system of superconducting flux qubits, as a function of time and of the number of qubits, in absence of interaction with the electron ensemble. The site dependent coupling constants of the qubit-qubit interaction was fixed at the values $J_{k, k^{\prime}}=$ $0.16 \times 2^{-\left|k^{\prime}-k\right|}[\mathrm{GHz}]$. Fig. 1 shows the behaviour of the Squeezing Parameter of Eq. (18), $\zeta_{q b, x^{\prime}}^{2}$. The pattern of the spin squeezing is the same for the different flux-qubit systems, that is spin squeezing appears at earlier times, it is destroyed at intermediate times and shows a revival at larger times, as the system approaches a stationary state. In all cases the stationary state of the system evolves in a squeezed spin state. This behaviour is consistent with the findings of [21]. The diagonal and the non-diagonal entries of the reduced density matrix, for the superconducting flux-qubits, as a function of time, are presented in Fig. 2 and in Fig. 3, respectively. As observed from the curves of Fig. 3, the non-diagonal entries of the reduced density matrix, though small, are not zero.

In Figs. 4-7, we show the effect of the coupling to the electron ensemble in a realistic case. As an example, we have taken $N_{q b}=3$ and $N_{S}=15$ electrons. We have fixed the value of the coupling constant on the interaction of the qubits and the electron spins to $g_{1}=g_{2}=g_{3}=0.6$ [GHz] [11]. For the qubit-qubit interaction we have taken $J_{1,2}=J_{2,1}=J_{2,3}=J_{3,2}=0.715, J_{1,3}=J_{3,1}=$ 0.482 [GHz] [34]. We have calculated the time evolution of an initial state consisting of a coherent state (16) for the electron sector, with $\theta_{S}=\Pi / 4$ and $\phi_{S}=0$, and of a state of the form $\mid N_{q b}=$ $3,\{1,1,1\}\rangle$ (Eq. (9)) for the qubits.

Fig. 4 shows the behaviour, as a function of time, of the squeezing parameter of the qubits, $\zeta_{q b, x^{\prime}}^{2}$, and of the electrons, $\zeta_{S, x^{\prime}}^{2}$. It can be seen from the Figure that the interaction with the electron spins 


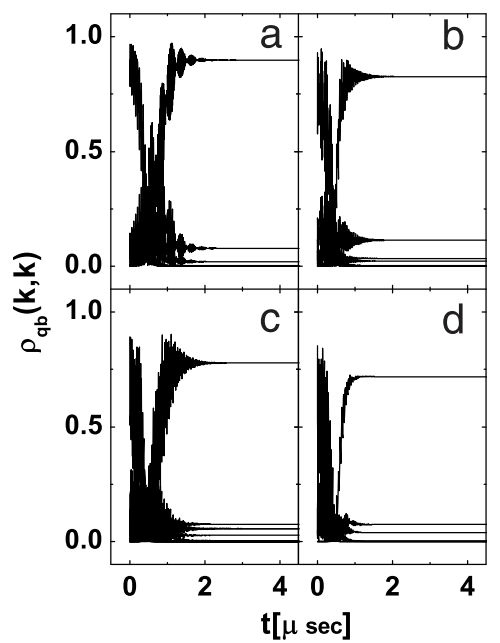

Fig. 2. Diagonal entries of the reduced density matrix, $\rho_{q b}(k, k)$, of the superconducting flux qubits, as a function of time. In Insets (a), (b), (c) and (d), we have displayed the results obtained for systems with $N_{q b}=3,4,5$ and 6 , respectively. The adopted parameters are those of Fig. 1.

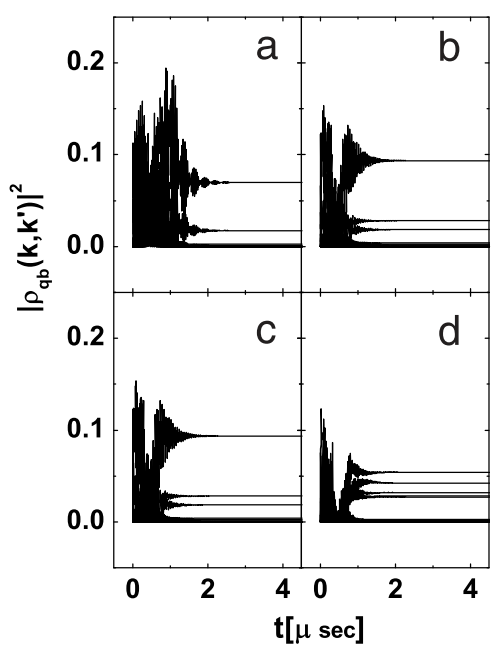

Fig. 3. Non-diagonal entries of the reduced density matrix, $\left|\rho_{q b}\left(k, k^{\prime}\right)\right|^{2}$, of the superconducting flux qubits, as a function of time. In Insets (a), (b), (c) and (d), we have displayed the results obtained for systems with $N_{q b}=3,4,5$ and 6 , respectively. The adopted parameters are those of Fig. 1.

reduced the squeezing effect in the flux qubit sector in the early and intermediate lapses of time, but the stationary value remains much the same that in the case of absence of coupling to the electrons. Also, the stationary state for the electron ensemble behaves as a squeezed state, $\zeta_{S, x^{\prime}}^{2}=0.94513$ in absence of coupling to the qubits, and $\zeta_{S, x^{\prime}}^{2}=0.94542$, when the interaction is turned on [21,23]. Fig. 5 shows the results obtained for the diagonal entries of the reduced density matrix of the two components of the hybrid system, $\rho_{q b}(k, k)$ and $\rho_{S}(k, k)$, as a function of time. The results have been obtained with the same set of parameters of the previous figure. The non-diagonal entries of the reduce density matrix, $\left|\rho_{q b}\left(k, k^{\prime}\right)\right|^{2}$ and $\left|\rho_{S}\left(k, k^{\prime}\right)\right|^{2}$, as a function of time, are shown in Fig. 6 . It can be observed from Figs. 5 and 6, that the interaction of the superconducting flux-qubits with the electrons favours the contribution of different states to the qubit reduced density matrix. Also, 

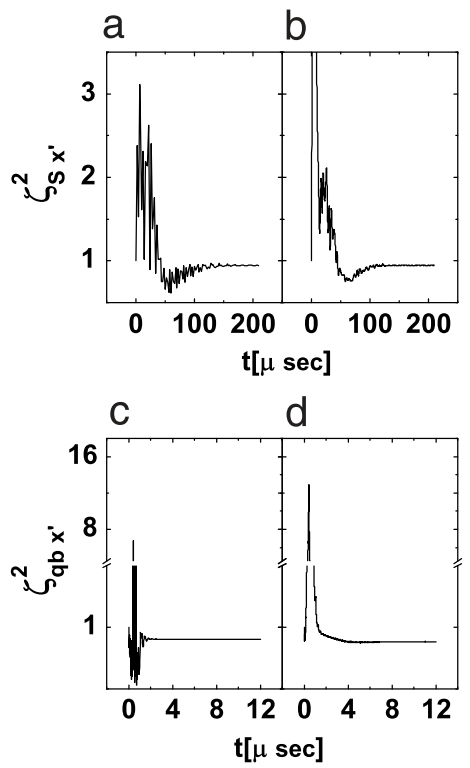

Fig. 4. Behaviour, as a function of time, of the electron ensemble squeezing parameter, $\zeta_{S, x^{\prime}}^{2}$, and of the superconducting flux qubits, $\zeta_{q b, x^{\prime}}^{2}$. The results obtained for the electron ensemble are displayed in Insets (a) and (b), while the results corresponding to the superconducting flux qubits are presented in Insets (c) and (d). The curves shown in Insets (a) and (c) have been computed in absence of coupling between the superconducting flux qubits and the electron ensemble. The flux qubits-electron interaction has been taken in account in Insets (b) and (d). For the electron sector we have fixed the coupling constants to the values $D=2.88[\mathrm{GHz}], E=0.15[\mathrm{GHz}]$ and $\gamma_{S}=2 \times 10^{-5}[\mathrm{GHz}]$. For the superconducting flux-qubits we have fixed the values of the coupling constants to $\Delta_{k}=2.40[\mathrm{GHz}], \epsilon_{k}=1.6[\mathrm{GHz}]$, and $\gamma_{q b}=4 \times 10^{-3}[\mathrm{GHz}]$. The coupling constants for the qubit-qubit interaction were fixed at $J_{1,2}=J_{2,1}=J_{2,3}=J_{3,2}=0.715, J_{1,3}=J_{3,1}=0.482[\mathrm{GHz}]$. For Insets (b) and (d), the flux qubits-electrons coupling constants were fixed at $g_{1}=g_{2}=g_{3}=0.6[\mathrm{GHz}]$.

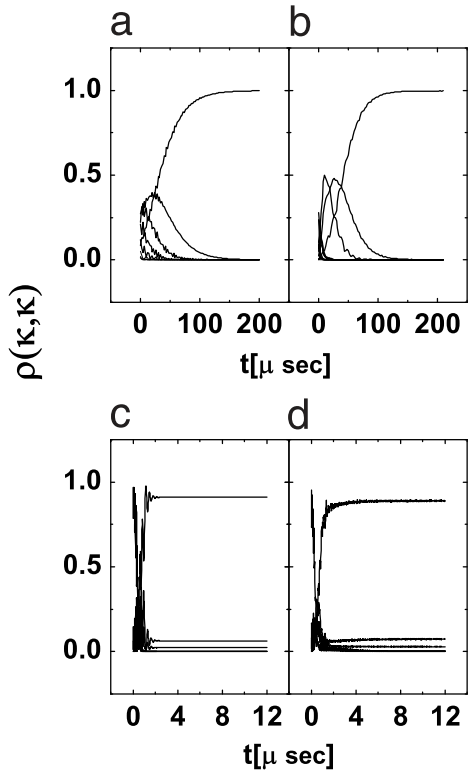

Fig. 5. Diagonal entries of the reduced density matrix of the spin electron ensemble, $\rho_{S}(k, k)$ (Insets (a) and (b)), and of the superconducting flux qubits, $\rho_{q b}(k, k)$ (Insets (c) and (d)), as a function of time. The adopted parameters are those of Fig. 5. 


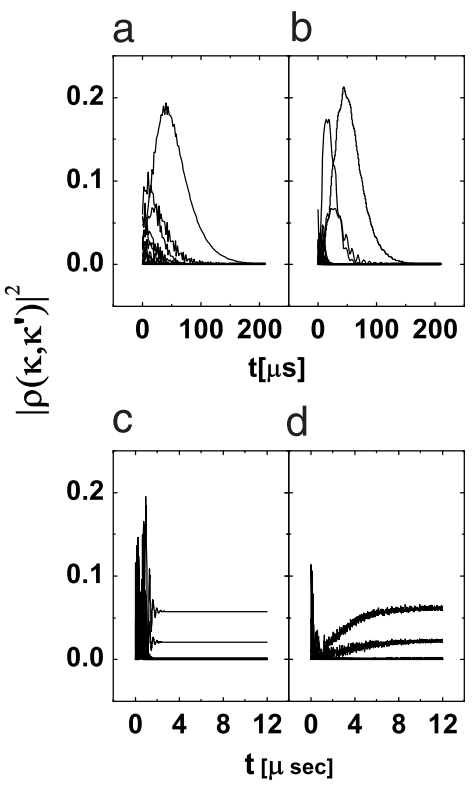

Fig. 6. Non-diagonal entries of the reduced density matrix of the electron ensemble, $\left|\rho_{S}\left(k, k^{\prime}\right)\right|^{2}$ (Insets (a) and (b)), and of the superconducting flux qubits, $\left|\rho_{q b}\left(k, k^{\prime}\right)\right|^{2}$ (Insets (c) and (d)), as a function of time. The adopted parameters are those of Fig. 5.
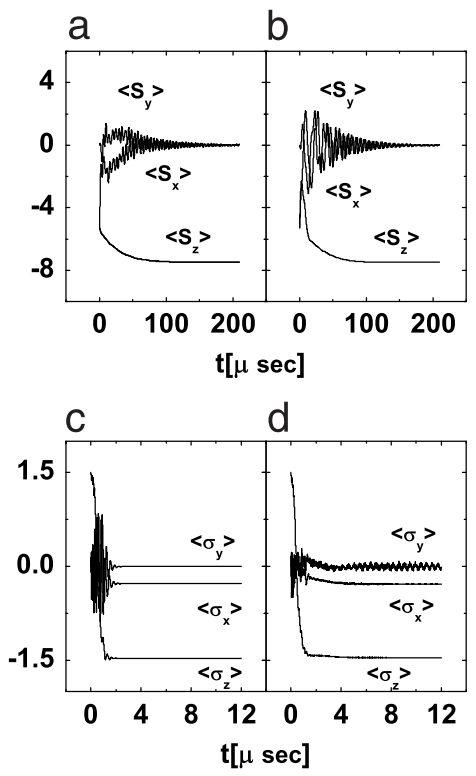

Fig. 7. Mean-value of the different components of the total spin of the superconducting flux qubits, $\left\langle\sigma_{k}\right\rangle$, and of the spin electron ensemble, $\left\langle S_{k}\right\rangle$, as a function of time. The adopted parameters are those of Fig. 5.

the coherence time of the superconducting flux-qubits in interaction with the electron ensemble is greater than in the case of absence of interaction. As a complementary information, we show in Fig. 7 the mean value of the components of the total spin, for both the superconducting flux qubits and for the electron ensemble. The results are presented in the same fashion of Figs. 4-6. 


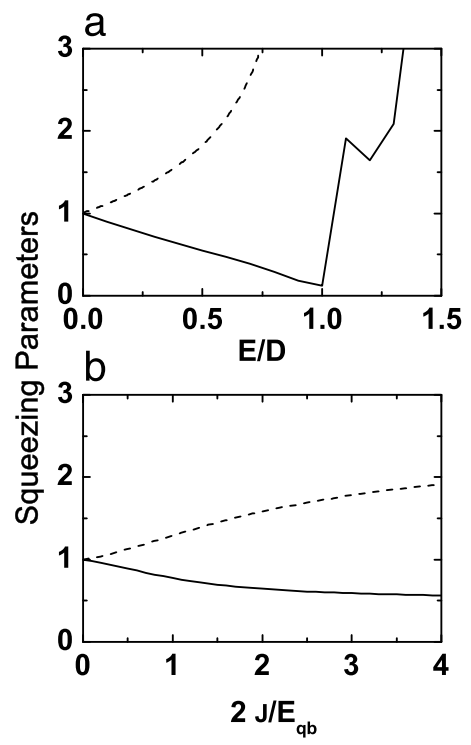

Fig. 8. Behaviour of the stationary limit of the squeezing parameter, in absence of coupling between the electron ensemble and the superconducting flux qubits, as a function of the coupling constants. In Inset (a), the results obtained for the squeezing parameters of the electron ensemble are displayed as a function of the relative value of the Lipkin coupling constant to the OAT coupling constant, $E / D$. In Inset (b), the results obtained for the squeezing parameters of the superconducting flux qubits are displayed as a function of the relative value of the site-site coupling constant to characteristic energy of the qubits, $J /\left(E_{q b} / 2\right)$. In both Insets, we draw with solid line the results for the $x^{\prime}$-direction squeezing parameters, and with dashed lines the $y^{\prime}$-direction. We have considered an electron ensemble with 15 spins, $N_{S}=15$, and a superconducting flux qubit system with $N_{q b}=3$. We have fixed the model parameters to the values $D=2.88[\mathrm{GHz}], \gamma_{s}=2 \times 10^{-5}[\mathrm{GHz}], \Delta_{k}=2.40[\mathrm{GHz}], \epsilon_{k}=1.6[\mathrm{GHz}], \gamma_{q b}=$ $4 \times 10^{-3}[\mathrm{GHz}]$ and $J_{k, k}=0[\mathrm{GHz}]$.

We have verified that these features persist when the number of components of the hybrid system increases.

Finally, we shall analyse the general dependence of the steady spin squeezed state on the different model parameters.

In Figs. 8 and 9, we study the properties of the stationary state of each subsystem, the electron ensemble and the superconducting flux qubits, in absence of interaction between them. Fig. 8 shows the stationary limit of the squeezing parameters as a function of the coupling constants. In Inset (a), the results obtained for the squeezing parameters of the electron ensemble are displayed as a function of the relative value of the Lipkin coupling constant to the OAT coupling constant, $E / D$. In Inset (b), the results obtained for the squeezing parameters of the superconducting flux qubits are displayed as a function of the relative value of the site-site coupling constant to characteristic energy of the qubits, $J /\left(E_{q b} / 2\right)$ (with $J\left(k, k^{\prime}\right)=J$ for $k \neq k^{\prime}$, and $J(k, k)=0$ ). In both Insets, we draw with solid line the results for the $x^{\prime}$-direction squeezing parameters, $\zeta_{x^{\prime}}^{2}$, and with dashed lines the $y^{\prime}$-direction, $\zeta_{y^{\prime}}^{2}$. We have considered an electron ensemble with 15 spins, $N_{S}=15$, and a superconducting flux qubit system with $N_{q b}=3$. The study of Fig. 8(a) shows the existence of two regions, concerning the squeezing properties of the electron ensemble. For $E / D<1$ the system is squeezed in the $x^{\prime}$-direction, while for $E / D>1$ the system is no longer squeezed. From Fig. 8(b) it can be concluded that the site-site interaction is responsible for the appearance of squeezing in the superconducting flux qubit system, and that it reaches a limiting value as $2 J / E_{q b}$ is increased. In Fig. 9, we have plotted the stationary value of the squeezing parameters as a function of the number of particles of each subsystem. Fig. 9(a) suggests that the value of the squeezing parameter for the electron ensemble is almost independent of the number of electron spins in the systems. Fig. 9(b) suggests that the increase in the number of flux-qubits tends to favour the squeezing of the system. 

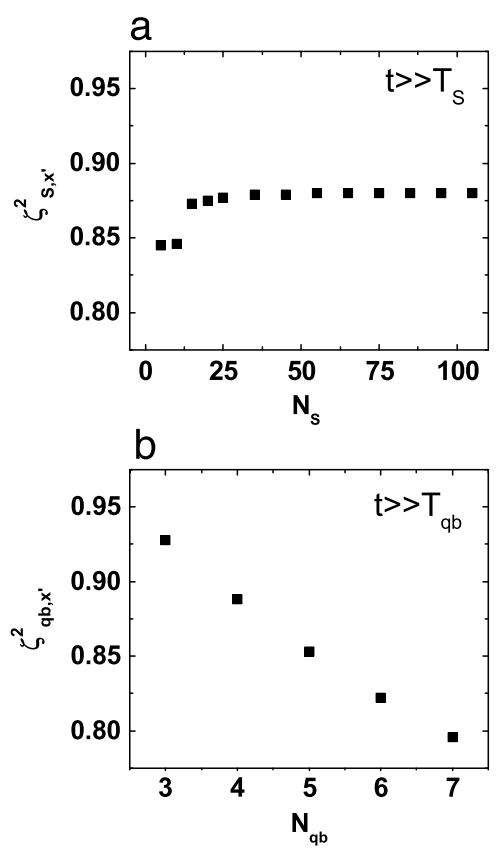

Fig. 9. Behaviour, in absence of coupling between the electron ensemble and the superconducting flux qubits, of the stationary limit of the squeezing parameter as a function of the number particles. In Inset (a), we display the results obtained for $\zeta_{x^{\prime}}^{2}$ as a function of the number of electron spins in the system $\left(t \approx 400[\mu \mathrm{s}] \gg T_{S}\right)$. In Inset $(\mathrm{b})$, we present the results obtained for $\zeta_{q b, x^{\prime}}^{2}$ as a function of the number superconducting flux qubits in the system $\left(t \approx 6[\mu \mathrm{s}] \gg T_{q b}\right)$. We have fixed model parameters to the values $D=2.88[\mathrm{GHz}], E=0.15[\mathrm{GHz}], \gamma_{s}=2 \times 10^{-5}[\mathrm{GHz}], \Delta_{k}=2.40[\mathrm{GHz}], \epsilon_{k}=1.6[\mathrm{GHz}], \gamma_{q b}=4 \times 10^{-3}[\mathrm{GHz}]$ and $J_{k, k^{\prime}}=0.5[\mathrm{GHz}]$, with $k \neq k^{\prime}$.

In Fig. 10, we present the results we have obtained for the dependence of the steady spin squeezing state on the characteristic coupling constants of the model, $E / D$ and $g / \sqrt{E_{q b} D}$, when the interaction between both subsystems is turned on. In Insets (a), (c) and (e), we show the behaviour of the squeezing parameter of the superconducting flux qubit system, $\zeta_{q b, x^{\prime}}^{2}$. In Insets (b), (d) and (f), the behaviour of the squeezing parameter of the electron ensemble, $\zeta_{S, x^{\prime}}^{2}$, is presented. The contour plots of insets (a) and (b) were calculated by fixing the site-site interaction of the qubits to the value $J\left(k, k^{\prime}\right)=0[\mathrm{GHz}]$. For Insets (c) and (d) we have fixed the site-site coupling constant to the value $J\left(k, k^{\prime}\right)=0.4[\mathrm{GHz}]$, while in Insets (e) and (f) the site-site coupling constant has been fixed to the value $J\left(k, k^{\prime}\right)=0.8[\mathrm{GHz}]$. We have adopted a hybrid system with $N_{S}=15$ electrons and $N_{q b}=3$ superfluid flux qubits. From Inset (a) it can be concluded that the interaction with the electron ensemble, even in absence of qubit-qubit interaction, can be used to drive the superconducting flux qubit system to a steady squeezed state. From insets (c) and (e) it can be seen that the increase in the site-site interaction among the qubits, enhances the superconducting flux qubits squeezing properties. Concerning the squeezing properties of the steady state of the electron ensemble, Insets (b), (d) and (f) strongly suggest the persistence of to well defined regions delimited by the relative values of the Lipkin coupling constant, $E / D$, and of the relative values of the coupling constant interaction with the superconducting flux qubits, $g / \sqrt{E_{q b} D}$. When the value of $g / \sqrt{E_{q b} D}$ is increased, lower values of the Lipkin constant, $E / D$, are needed to obtained the same value of the squeezing electron ensemble parameter. If the site-site superconducting flux qubits interaction is switched on, this effect is enhanced.

As reported in $[21,22]$ non-Hermitian dynamics can amplify the entanglement and spin squeezing properties of the corresponding systems. This is a relevant feature, because the achievement of squeezing of a canonical variable, $\zeta_{x^{\prime}}^{2}$, implies that quantum memory and metrology applications gain 


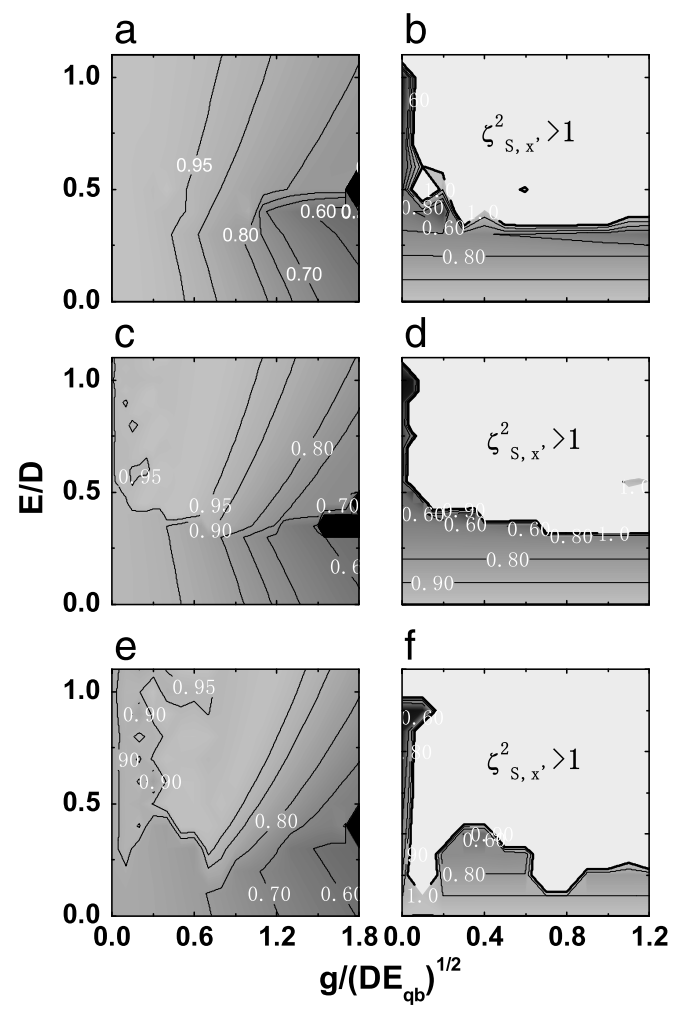

Fig. 10. Behaviour of the stationary value in the $x^{\prime}$ direction of the squeezing parameter as a function of the characteristic coupling constants of the hybrid system, $E / D$ and $g / \sqrt{E_{q b} D}$. In Insets (a), (c) and (e), we show the contour plot of $\zeta_{q b, x^{\prime}}^{2}$ obtained for the superconducting flux qubit system. In Insets (b), (d) and (f), we show the contour plot of $\zeta_{x^{\prime}}^{2}$ obtained for the electron ensemble system. Insets (a) and (b) were calculated by fixing the site-site interaction of the qubits to the value $J\left(k, k^{\prime}\right)=0[\mathrm{GHz}]$. For Insets (c) and (d) we have fixed the site-site coupling constant to the value $J\left(k, k^{\prime}\right)=0.4[\mathrm{GHz}]$. While for Insets (e) and (f) the site-site coupling constant was fixed to the value $J\left(k, k^{\prime}\right)=0.8[\mathrm{GHz}$. We have adopted a hybrid system with $N_{S}=15$ electrons and $N_{q b}=3$ superfluid flux qubits. We have fixed the model parameters to the values $D=2.88[\mathrm{GHz}], \gamma_{S}=2 \times 10^{-5}[\mathrm{GHz}], \Delta_{k}=2.40[\mathrm{GHz}], \epsilon_{k}=1.6[\mathrm{GHz}]$ and $\gamma_{q b}=4 \times 10^{-3}[\mathrm{GHz}]$.

by an increased signal-to-noise ratio $[25,43]$, i.e. $\zeta^{2} \simeq 0.5$ gives an enhancement in the signal-to-noise ratio of $\simeq 3[\mathrm{~dB}]$.

In this work, we have taken into account dissipative effects through an effective non-Hermitian Hamiltonian [49], and we have adopted the time evolution formalism presented in [50]. Similar results, concerning the generation of spin squeezing, have been reported in [43]. The Authors of [43] have studied, by using the master equation formalism of Reiter and Sørensen [54], the implementation of hybrid-systems to enhance spin squeezing by using a highly dissipative ancillary system. In [43] the master equation for the reduced density matrix of spins has computed following the prescription of [54]. The Authors of [54] have shown that their master equation formalism reduces to the Feshbach projection formalism [49,50], if quantum jumps [55-57] are not taken into account.

\section{Conclusions}

In this work we have studied the time evolution of the spin-squeezing of an array of superconducting flux-qubits coupled to an ensemble of electrons. We have analysed the spinsqueezing properties of both components of the hybrid system as well as the induced environmentdecoherence. The electron-component of the system was modelled by a one-twist Hamiltonian [29] 
plus a Lipkin-type interaction [27]. The electron-qubits interaction has been diagonalized by a convenient choice of the basis. It is shown that both the superconducting flux qubits and the electron ensemble evolves to a stationary squeezed state. The interaction of the superconducting flux qubits with the electron ensemble affects the time-dependent pattern of the spin-observables and of the entries of the reduced density matrix. This result may add to the results reported in [14], where a similar hybrid system was analysed in terms of solutions of a Jaynes-Cummings type of Hamiltonian.

\section{Acknowledgements}

This work was partially supported by the National Research Council of Argentine (CONICET, PIP 112-2008-01-00282) and by the Agencia Nacional de Promocion Cientifica (ANPCYT, PICT11-0300113) of Argentina. M.R. and O.C. are members of the scientific research career of the CONICET.

\section{References}

[1] A.C. Wilson, et al., Nature 512 (2014) 57.

[2] T. van der Sar, et al., Nature 484 (2012) 82.

[3] S.K. Mishra, L. Chotorlishvili, A.R.P. Rau, J. Berakdar, Phys. Rev. A 90 (2014) 033817.

[4] P.T. Greenland, et al., Nature 465 (2010) 1057.

[5] E. Togan, et al., Nature 466 (2010) 730.

[6] P.R. Eastham, P. Kirton, H.M. Cammack, B.W. Lovett, J. Keeling, Phys. Rev. A 94 (2016) 012110.

[7] A.N. Vamivakas, M. Atatüre, Contemp. Phys. 51 (2010) 17.

[8] P.R. Eastham, A.O. Spracklen, J. Keeling, Phys. Rev. B 87 (2013) 195306.

[9] D. Boyanovsky, Phys. Rev. A 87 (2013) 033815.

[10] Ze-Liang Xiang, Sahel Ashhab, J.Q. You, F. Nori, Rev. Modern Phys. 85 (2013) 623.

[11] X. Zhu, et al., Nature 478 (2011) 221.

[12] Xin-You Lü, Ze-Liang Xiang, Wei Cui, J.Q. You, Franco Nori, Phys. Rev. A 88 (2013) 012329.

[13] N. Metwally, M.R.B. Wahiddin, M. Bourennane, Opt. Commun. 257 (2006) 206.

[14] T. Hümmer, G.M. Reuther, P. Hänggi, D. Zueco, Phys. Rev. A 85 (2012) 052320.

[15] Yueyin Qiu, Wei Xiong, Lin Tian, J.Q. You, Phys. Rev. A 89 (2014) 042321.

[16] M. Schlosshauer, Rev. Modern Phys. 76 (2004) 1267 and references therein.

[17] W.H. Zurek, Phys. Rev. D 26 (1982) 1862; W.H. Zurek, Phys. Rev. D 24 (1981) 1516;

F.M. Cucchietti, J.P. Paz, W.H. Zurek, Phys. Rev. A 72 (2005) 052113;

W.H. Zurek, Prog. Math. Phys. 48 (2007) 1;

B. Damski, H.T. Quan, W.H. Zurek, Phys. Rev. A 83 (2011) 062104.

[18] J. Ma, X. Wang, C.P. Sun, F. Nori, Phys. Rep. 509 (2011) 89.

[19] Zhe Sun, Phys. Rev. A 84 (2011) 052307.

[20] V.V. Dodonov, C. Valverde, L.S. Souza, B. Baseia, Ann. Physics 37 (2016) 296.

[21] Tony E. Lee, Florentin Reiter, Nimrod Moiseyev, Phys. Rev. Lett. 113 (2014) 250401.

[22] Ling-Na Wu, Guang-Ri Jin, L. You, Phys. Rev. A 92 (2015) 033826.

[23] Yong-Hong Ma, Xue-Feng Zhang, Phys. Rev. B 89 (2014) 144113.

[24] Yong-Hong Ma, Xue-Feng Zhang, Jie Song, E. Wu, Ann. Physics 369 (2016) 36.

[25] Yuichiro Matsuzaki, Xiaobo Zhu, Kosuke Kakuyanagi, Hiraku Toida, Takaaki Shimo-Oka, Norikazu Mizuochi, Kae Nemoto, Kouichi Semba, William J. Munro, Hiroshi Yamaguchi, Shiro Saito, Phys. Rev. Lett. 114 (2015) 120501.

[26] T. Fernholz, H. Krauter, K. Jensen, J.F. Sherson, A.S. Sørensen, E.S. Polzik, Phys. Rev. Lett. 101 (2008) 073601.

[27] H.J. Lipkin, N. Meshkov, A.J. Glick, Nuclear Phys. 62 (1965) 188.

[28] P. Ring, P. Schuck, The Nuclear Many Body Problem, Springer Verlag, Berlin, Heidelberg, 1980.

[29] M. Kitagawa, M. Ueda, Phys. Rev. A 47 (1993) 5138.

[30] N. Mizuochi, P. Neumann, F. Rempp, J. Beck, V. Jacques, P. Siyushev, K. Nakamura, D.J. Twitchen, H. Watanabe, S. Yamasaki, F. Jelezko, J. Wrachtrup, Phys. Rev. B 80 (2009) 041201(R).

[31] P.L. Stanwix, L.M. Pham, J.R. Maze, D. Le Sage, T.K. Yeung, P. Cappellaro, P.R. Hemmer, A. Yacoby, M.D. Lukin, R.L. Walsworth, Phys. Rev. B 82 (2010) 201201(R).

[32] D. Farfurnik, A. Jarmola, L.M. Pham, Z.H. Wang, V.V. Dobrovitski, R.L. Walsworth, D. Budker, N. Bar-Hill, Phys. Rev. B 92 (2015) 201201(R).

[33] S.H.W. van der Ploeg, et al., Phys. Rev. Lett. 98 (2007) 057004.

[34] A.O. Niskanen, et al., Science 316 (2007) 723.

[35] T. Hime, et al., Science 314 (2006) 1427.

[36] M. Stern, G. Catelani, Y. Kubo, C. Grezes, A. Bienfait, D. Vion, D. Esteve, P. Bertet, Phys. Rev. Lett. 113 (2014) 123601.

[37] Zheng-Yuan Xue, et al., Quantum Inf. Process. 15 (2016) 721.

[38] Huang Wen, Zou Xu-Bo, Guo Guang, Chin. Phys. B 24 (2015) 064207.

[39] D. Marcos, M. Wubs, J.M. Taylor, R. Aguado, M.D. Lukin, A.S. Sørensen, Phys. Rev. Lett. 105 (2010) 210501.

[40] T.P. Orlando, J.E. Mooij, Lin Tian, Caspar H. van der Wal, L.S. Levitov, Seth Lloyd, J.J. Mazo, Phys. Rev. B 60 (1999) 15398.

[41] B.L.T. Plourde, J. Zhang, K.B. Whaley, F.K. Wilhelm, T.L. Robertson, T. Hime, S. Linzen, P.A. Reichardt, C.-E. Wu, John Clarke, Phys. Rev. B 70 (2004) 140501.

[42] A. Ballesteros, O. Civitarese, F.J. Herranz, M. Reboiro, Phys. Rev. B 68 (2003) 214519. 
[43] Shane Dooley, Emi Yukawa, Yuichiro Matsuzaki, George C. Knee, William J. Munro, Kae Nemoto, New J. Phys. 18 (2016) 053011.

[44] S.D. Bennett, N.Y. Yao, J. Otterbach, P. Zoller, P. Rabl, M.D. Lukin, Phys. Rev. Lett. 110 (2013) 156402.

[45] M.W. Doherty, F. Dolde, H. Fedder, F. Jelezko, J. Wrachtrup, N.B. Manson, L.C.L. Hollenberg, Phys. Rev. B 85 (2012) 205203.

[46] J.M. Taylor, P. Capperallo, L. Childress, L. Jiang, D. Budker, P.R. Hemmer, A. Yacoby, R. Walsworth, M.D. Lukin, Nat. Phys. 4 (2008) 810 .

[47] O. Civitarese, M. Reboiro, L. Rebón, D. Tielas, Phys. Lett. A 374 (2010) 424.

[48] O. Civitarese, M. Reboiro, L. Rebón, D. Tielas, Phys. Lett. A 374 (2010) 2117;

O. Civitarese, M. Reboiro, L. Rebón, D. Tielas, Phys. Lett. A 373 (2009) 754.

[49] H. Feshbach, Ann. Physics 5 (1958) 357.

[50] I. Rotter, J. Phys. A 42 (2009) 153001;

I. Rotter, J.P. Bird, Rep. Progr. Phys. 78 (2015) 114001.

[51] O. Civitarese, M. Reboiro, Phys. Lett. A 357 (2006) 224;

M. Reboiro, O. Civitarese, L. Rebón, Phys. Lett. A 366 (2007) 241.

[52] K.T. Hecht, in: H. Araki, et al. (Eds.), Lecture Notes in Physics, Vol. 290, Springer-Verlag, Berlin, 1980.

[53] A. Luis, N. Korolkova, Phys. Rev. A 74 (2006) 043817.

[54] Florentin Reiter, Anders S. Sørensen, Phys. Rev. A 85 (2012) 032111

Florentin Reiter, Michael J. Kastoryano, Anders S. Sørensen, New J. Phys. 14 (2012) 053022.

[55] P. Zoller, M. Marte, D.F. Walls, Phys. Rev. A 35 (1987) 198

[56] J. Dalibard, Y. Castin, K. Mølmer, Phys. Rev. Lett. 68 (1992) 580.

[57] M.B. Plenio, P.L. Knight, Rev. Modern Phys. 70 (1998) 101. 